\title{
POLÍTICAS ENERGÉTICAS MUNDIAIS SOBRE O USO DA ENERGIA SOLAR
}

\section{GLOBAL ENERGY POLICIES ABOUT THE USE OF SOLAR ENERGY}

\author{
Adriana de Souza Nascimento ${ }^{1}$; Ricardo Moreira da Silva²; Eufrásio Vieira dos Anjos Júnior ${ }^{3}$ \\ ${ }^{1}$ Universidade Federal da Paraíba - UFPB - João Pessoa - Brasil \\ adriana.souza.adm@ hotmail.com \\ 2Universidade Federal da Paraíba - UFPB - João Pessoa - Brasil \\ ricardomoreira0203@hotmail.com \\ 3Universidade Federal da Paraíba - UFPB - João Pessoa - Brasil \\ eufrasiovieira@hotmail.com
}

\begin{abstract}
Resumo
Com intuito de reduzir a dependência dos combustíveis fósseis, minimizar os impactos ambientais e incentivar um novo desenvolvimento industrial, o setor de energia tem provocado mudanças estratégicas no que diz respeito a sua política. O objetivo deste artigo é expor uma revisão das diferentes políticas energéticas sobre o uso de energia solar, em diferentes países no mundo. A metodologia utilizada neste estudo foi à pesquisa bibliográfica, o que permitiu a realização de uma análise comparativa a partir de um amplo trabalho de pesquisa. A política energética é uma estratégia que os governos abordam as questões de desenvolvimento de energia juntamente com o consumo da indústria para sustentar seu crescimento, incluindo sua distribuição, produção $e$ consumo; Neste contexto, a demanda mundial de energia primária é projetada em um cenário para expandir em quase 60\% entre 2002 a 2030. Logo, as tarifas FIT, isenção de impostos e outros tipos de subsídios determinados pelo poder público, são as políticas mais utilizadas nos países emergentes, visto que, melhoram a qualidade do meio ambiente, proporcionam motivação, interesse significativo para o desenvolvimento e uso da tecnologia das energias renováveis, político, institucional e, sobretudo o desenvolvimento econômico.
\end{abstract}

Palavras-chave: energia solar; políticas energéticas; energia solar fotovoltaica.

\section{Introdução}

A adoção de energias alternativas tem sido amplamente procurada desde o inicio da década de 70. Com a crise do petróleo o mundo começou a procurar segurança no fornecimento energético e na redução da dependência da importação de energia. Juntamente com a crise, as preocupações ambientais fizeram com que a procura de fontes alternativas e mais limpas de produção de energia fossem ampliadas e dentre essas alternativas, a energia solar é uma das que vem apresentando significativa atenção. 
A energia solar é uma das fontes de energia mais limpas e que não faz comprometer ou adicionar o aquecimento global. "O sol é a fonte de energia renovável e o aproveitamento dessa energia tanto como fonte de calor quanto de luz, é uma das alternativas energéticas mais promissoras para enfrentarmos os desafios do novo milênio" (DYSON, 2001). Pertencente ao grupo chamado "Outras Fontes" (ou "fontes alternativas") que, de 1973 a 2006, aumentou em 500\% a sua participação na matriz energética mundial. (ANEEL, 2008).

Atualmente é ampla a preocupação com uma futura falta de energia para a humanidade, bem como os impactos ambientais de muitas formas de captação da mesma; Sua disponibilidade é acessível, abundante, corretamente ambiental e com mínimos riscos ecológicos associados à produção e uso, sendo um dos fatores importantes para a melhoria desejada na qualidade de vida das pessoas.

Segundo Hasnain (1995) “a crescente escassez de combustíveis fósseis tem despertado o interesse mundial no aproveitamento da energia solar".

\begin{abstract}
As atuais fontes de energia mais utilizadas provem do meio ambiente e são esgotáveis, sua produção é dividida entre fontes poluentes e fontes limpas. As fontes limpas não causam dano à natureza, existe em quantidade disponível para o uso, possuindo um custo aceitável e sem causar impactos negativos à sociedade. Uma fonte limpa, busca a minimização das perdas e danos, seja na quantidade de resíduo, no impacto do meio ambiente, no representativo financeiro ou no impacto para a sociedade (SILVA, 2011).
\end{abstract}

Fatores estes, tornam as fontes poluentes cada vez mais mal vistas; comprovando que nos últimos anos a energia fotovoltaica tem sido vista internacionalmente como uma tecnologia bastante promissora. Experiências internacionais apresentam importantes contribuições para análise sobre expansão do mercado, ganhos na escala de produção e redução de custos para os investidores (VIANA, 2011).

Com grande potencial futuro, embora no presente, compreenda meramente uma parte da demanda da energia global, ou seja, $0,05 \%$ da oferta total de energia primária; A produção de energia solar através das células fotovoltaicas pode gerar cerca de menos de $1 \%$ da oferta total de eletricidade, isto devido à energia solar ser ainda considerada o tipo de energia renovável mais cara (EREC, 2005).

Todavia, vem experimentando um forte progresso tecnológico no mundo nos últimos anos, acentuado a partir de 2004, esse desenvolvimento é reflexo do crescimento exponencial dos volumes de produção e de instalação FV e na redução do custo dessa fonte, ocasionada pelos programas de incentivos para a geração fotovoltaica no mundo (A CARTA DO SOL, 2011).

"Os sistemas energéticos mundiais são altamente dependentes de fontes fósseis, pois no passado essas fontes eram abundantes e com preço atraente" (SCHETTINO, 2013). Desde a crise da década de 1970, que isso não é mais verdade, destaca Battaglini et al (2009), pois está havendo um forte aumento da demanda energética em função do grande crescimento econômico (com estimativa de crescimento da demanda energética mundial em 60\%, até 2030), o que demonstra que os 
sistemas de energia (geração e consumo) precisam mudar para acompanhar esse crescimento da economia.

Contudo, tem-se observado um grande crescimento no seu desenvolvimento e instalações; Na Tabela 1, observam-se projeções futurais que chegam até meados do ano 2030.

Tabela 1- Desenvolvimento e instalação de energia solar fotovoltaica em vários países

\begin{tabular}{ccccc}
\hline Ano & EUA $(\mathbf{M W})$ & EUROPA $(\mathbf{M W})$ & JAPÃO (MW) & $\begin{array}{c}\text { EM TODO O } \\
\text { MUNDO (MW) }\end{array}$ \\
\hline $\mathbf{2 0 0 0}$ & 140 & 150 & 250 & 1000 \\
$\mathbf{2 0 1 0}$ & 3000 & 3000 & 5000 & 14000 \\
$\mathbf{2 0 2 0}$ & 15000 & 15000 & 30000 & 70000 \\
$\mathbf{2 0 3 0}$ & 25000 & 30000 & 72000 & 140000 \\
\hline
\end{tabular}

Fonte: Adaptado de Solangi et al (2011)

Este tipo de energia reduz as linhas de transmissão de redes elétricas tradicionais, não emite gases tóxicos na atmosfera, não esgota os recursos naturais, aumenta a independência energética nacional/regional, além de diversificar e assegurar o abastecimento energético. Portanto, "a energia solar tem se mostrado ambientalmente vantajosa a qualquer outra fonte de energia, tornando-se assim, a peça fundamental a qualquer programa de desenvolvimento sustentável" (TSOUTOS, 2005).

\section{2. $O$ consumo global de energia}

A demanda mundial de energia primária é projetada em um cenário para expandir em quase $60 \%$ entre 2002 a 2030, tendo cerca de um aumento anual médio de 1,7\% por ano. Segundo pesquisas, chegará ao equivalente a 16,5 bilhões de toneladas de petróleo em comparação com 10,3 bilhões em 2002. Entretanto, os combustíveis fósseis ainda continuarão a dominar o uso de energia global até meados de 2025.

Documentos internacionais reportam para o ano de 2050 que $50 \%$ da geração de energia no mundo viram de fontes renováveis. Dessa demanda, $25 \%$ serão supridos pela energia solar fotovoltaica. Populações do fim do século dependerão em ate $90 \%$ das renováveis, dos quais $70 \%$ será de fotovoltaica.

Pesquisas afirmam que os combustíveis fosseis ainda serão responsáveis por cerca de $85 \%$ do aumento da demanda primária no mundo inteiro. Sua participação na demanda total vai aumentar ligeiramente, de $80 \%$ em 2002 para $82 \%$ em 2030 e quando comparado com o todo, a participação das fontes renováveis de energia aumentará discretamente, em torno de $4 \%$, enquanto que a energia nuclear vai cair de $7 \%$ para $5 \%$ (IEA, 2008).

Ainda segundo IEA (2008) “o petróleo continuará a ser o maior combustível no mix global de energia primária, apesar de sua participação decair de 36\% em 2002 para 35\% em 2030", pesquisas recentes realizadas pelo relatório anual WEO (2012), afirmam que "a necessidade de eletricidade nas economias emergentes impulsionará um aumento de quase $70 \%$ em todo o mundo". Para melhor visualização, segue Figura 1, que esboça uma projeção desde o ano 2010 até 2035. 
Figura 1 - Projeção global de geração de energia (2010 - 2035)

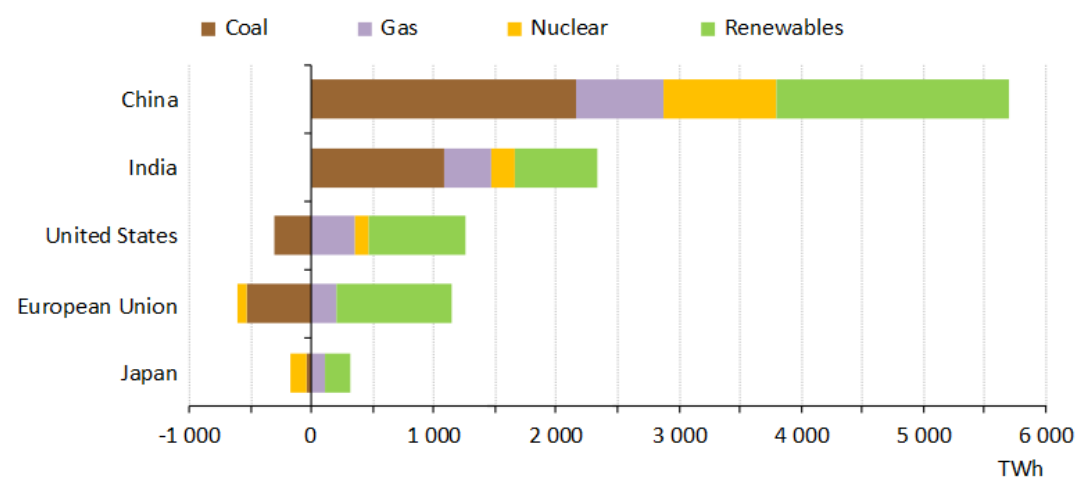

Fonte: WEO, 2012

\subsection{Políticas energéticas}

De acordo com Elkins (2004), "não existe um modelo ideal e universal de política pública de uso de energias renováveis, provavelmente ela se adequará ao contexto de sua história e cultura".

A política energética é uma estratégia em que os governos decidem abordar as questões de desenvolvimento de energia juntamente com o consumo da indústria para sustentar seu crescimento, incluindo a distribuição de energia, produção e consumo; Os atributos dessa política podem incluir legislação, tratados internacionais e incentivos ao investimento, logo, desempenha um papel vital para mitigar os impactos do aquecimento mundial e crise de disponibilidade da demanda de energia (EPD, 2013).

A busca da redução dos impactos ambientais no setor de energia tem provocado mudanças nos governos e a utilização de estratégias com intuito de reduzir a dependência dos combustíveis fósseis e incentivar um novo desenvolvimento industrial (SILVA, 2011).

Uma variedade de políticas como tarifa FIT (Feed in tariff, que se trata de uma obrigação por parte de uma concessionária, quando ela é obrigada a comprar eletricidade gerada por produtores de energias renováveis em sua área de atuação); créditos fiscais; leis de preços especiais; Incentivos à produção, selos verdes; ou seja, "há todo um arcabouço de exigência de quotas e sistemas de negociação que foram desenvolvidos e implementados para promover o uso de energias renováveis" (KISSEL, 2006).

De acordo com Elkins (2004), "não existe um modelo ideal e universal de política pública de uso de energias renováveis, provavelmente ela se adequará ao contexto de sua história e cultura”. Neste trabalho, são brevemente apresentadas políticas energéticas de uso da energia solar em diversos países, formando um panorama mundial de seu uso.

\section{Metodologia}

Este trabalho caracteriza-se por pesquisa bibliográfica por se tratar de uma revisão da literatura já existente sobre a energia solar, o que permitiu a realização de uma análise comparativa e a 
exposição de diferentes políticas energéticas sobre o uso desta fonte de energia renovável, em diferentes países no mundo.

Países como EUA, Canadá, Espanha, Alemanha, França, China, Austrália, Paquistão e Malásia foram abordados, de maneira análoga, a partir de um amplo trabalho de pesquisa em material já publicado, através de livros, artigos periódicos e até mesmo através da rede. Segundo Miguel (2010) "uma abordagem metodológica adequada de pesquisa compreende diferentes níveis de abrangência e profundidade".

\section{Resultados e discussões}

\subsection{EUA}

O país adotou mecanismos de padrão de renovação (RPS) em seus 28 estados. É exigida uma percentagem de energias renováveis a energia produzida, como solar, eólica, geotérmica e biomassa.

A maioria dos objetivos políticos visa facilitar a diversificação da geração de eletricidade, reduzir a dependência do estado em relação aos combustíveis fósseis, bem como, aumentar a implantação de energias renováveis e assim, diminuir a redução das emissões de carbono no meio ambiente (CARLEY, 2009).

Em meados de 1978, foi adotado pela primeira vez o PURPA (Regulador Político), que, trabalhava desde então a inserção de energias alternativas em resposta ao aumento do custo das energias fósseis ao longo da década de 70. De 1981 até 1990 foram instalados cerca de 12 GW no país com esta forma de incentivo; Além disso, foram criados os primeiros créditos fiscais de investimento para tecnologias em energias alternativas distribuídas, beneficiando assim produtor residencial e empresarial.

Hoje, "o setor energético solar nos EUA incentiva durante mais de 8 anos em crédito fiscal de investimento" (MENDONÇA, 2009). Milhões de americanos estão aproveitando os benefícios de possuir um sistema distribuído de gerar energia através da energia solar. Novamente Mendonça (2009), assegura em recente relatório sobre o impacto econômico da política do investimento dos créditos fiscais, que a "utilização deste incentivo do governo pode resultar em mais de $6000 \mathrm{MW}$ anuais de energia solar fotovoltaica e mais instalações solares térmicas até 2016".

\subsection{Canadá}

O setor de energia é uma parte importante da economia do país em termos de investimento, comércio, geração de renda e emprego. Desde 1970, o país formulou várias medidas estratégias para acelerar o desenvolvimento de sistemas de eficiência energética e tecnologia de energias renováveis e tem feito progressos significativos; "De 1990 a 2003 a eficiência energética melhorou 
cerca de $13 \%$ só em 2003, reduziu as emissões de gases de efeito estufa em 52,3 megatons" (LIMING, 2008).

O governo possui um papel de grande relevância no desempenho e incentivo do uso da energia solar. A tarifa FIT, é a política de incentivo mais eficaz do programa ao uso de fontes de energia renováveis, tanto é que, está previsto um aumento a ponto de que em toda província instale em seus telhados uma estrutura de energia solar. A mudança mais notável em relação a crescimento foi a produção de painéis fotovoltaicos ter crescido $123 \%$ em 2008.

Infelizmente, a matriz energética do país ainda é forte no uso do carvão. "Apenas a cidade de Ontário produz cerca de 28 Twh/ano de energia a partir do carvão; evidentemente para eliminar todo o carvão nos próximos 5 anos, seria necessário um poderoso investimento em energias renováveis" (CEA, 2009) e isso dificilmente irá acontecer.

É de interesse econômico do governo federal canadense que as políticas públicas subsidiem a produção, tecnologia e importação dos painéis, por tal motivo, o governo está melhorando e adaptando o setor de processamento de fabricação com as novas politicas de tributação, energia, comércio, trabalho, pesquisa, infraestrutura, pesquisa, desenvolvimento, entre outros; Além de possuir uma força de trabalho qualificada e compromisso do governo com inovação e crescimento econômico, tal política proporcionará benefícios econômicos, ambientais e sociais significativos (SARAH, 2009).

\subsection{Alemanha}

A Alemanha é a líder mundial na instalação de sistemas de energia solar fotovoltaica com uma "capacidade aproximada de $3.8 \mathrm{GW}$ (dados de 2007), mesmo assim, esse tipo de produção é estimado apenas em 4\%" (IEA, 2008).

O governo investe forte na utilização deste tipo de energia, incentivando o uso através de
uma remuneração constante para a energia produzida. O país tem quase a mesma
capacidade de geração de energia solar que todas as outras nações do mundo juntas. Além
disso, o país pretende, até 2020 , cortar em $40 \%$ as emissões de gases estufa. Assim, calcula-
se que o investimento para o sistema fotovoltaico entre 2009 e 2021 seja de
aproximadamente 30 bilhões de euros (BHANDARI, 2009).

Segundo EPE (2012) o governo alemão assegura tarifas-premio favoráveis ao investimento pelos consumidores nessas instalações. Os subsídios ao investimento existem em diferentes regiões da Alemanha e se tornam cada vez mais atraentes ao consumidor. A meta da indústria solar alemã é incrementar o uso de baterias para o seu armazenamento, em contrapartida, o consumidor alemão terá que pagar mais pela energia elétrica em 2013. "Para financiar o custo da mudança energética do país para uma matriz renovável, o preço de cada quilowatt-hora passou de 3,59 para 5,30 centavos de euro" (DW BRASIL, 2013).

\subsection{Espanha}


MIDIC (2007) já afirmava: “o governo cada vez mais busca, além da adequação aos padrões da UE e às metas de redução da emissão de $\mathrm{CO} 2$, reduzir a dependência a importações de fontes energéticas não renováveis". Conforme EPE (2012) no ano de 2010, o governo espanhol lançou o plano nacional para energias renováveis 2011-2020 (PANER) que estabelece que cerca de 3,6\% da demanda total de eletricidade na Espanha em 2020 devera ser atendida por geração solar fotovoltaica.

Segundo Luigi (2010), "o ano de 2008 foi um ano muito importante para o país; O governo estabeleceu um novo marco regulatório a fim de introduzir novas regras para instigar o aumento do mercado de células fotovoltaicas incentivando assim, ainda mais o uso de energias renováveis".

O país é um dos líderes mundiais no que se refere à energia eólica, sendo a tecnologia renovável mais desenvolvida no país. No que se refere a energia solar, ela é provida de instalações de grande escala, possuindo as chamadas "fazendas solares", o fato se explica pois o país hispânico é mais provido de radiação solar do que qualquer outro país europeu, por isso no ano de 2008 foi marcado com uma capacidade instalada de mais de $3500 \mathrm{MW} \mathrm{e}$ de acordo com a lei 35/2006 o governo estabeleceu um desconto de imposto de 6\% (2008), $4 \%$ (2009), 2\%(2010), dos benefícios anuais do sistema fotovoltaico (LUIGI, 2010). Fato este, que confirma ainda mais o seu crescimento.

\subsection{França}

A França está em quinto lugar na produção de energia solar fotovoltaica, a tarifa FIT é uma das principais políticas adotadas para o sistema fotovoltaico, seguido de incentivos do governo como empréstimos verdes, incentivos fiscais, entre outros.

No que se refere ao mercado de renováveis, esse país em 2009, gerou uma receita de US\$ 9,5 bilhões, o que representou uma taxa de crescimento de $2,7 \%$ entre 2005 e 2009 . No entanto, é importante destacar que, exatamente no último período analisado (2008-2009), as taxas foram decrescentes e também se espera que esse decréscimo seja continuado até 2014 (ADEME, 2008).

Segundo Dusonchet (2010), “toda coordenação das políticas de energias renováveis no país, provem do ministério da agricultura e sua capacidade instalada acumulada em energia solar fotovoltaica deverá aumentar a partir de 2012 de 1,1GW a 5,4 GW”.

\subsection{China}

O crescimento econômico da China nas últimas duas décadas foi desenfreado, o que implicou a emissões de carbono no meio ambiente; "O país alcançou em 2002 a posição de segundo maior país consumidor de petróleo no mundo, ficando atrás apenas dos EUA e superando o Japão que, como todos os países industrializados, têm registrado, nas últimas décadas, um relativo aumento no consumo" (SOUZA, 2006). Diante disto, fez-se necessário a utilização e aplicação do sistema de energia solar, trazendo então grandes benefícios ambientais e econômicos.

Hoje, "o potencial de energia solar na China é muito alto onde centenas de fábricas produzem milhões de equipamentos fotovoltaicos nos últimos cinco anos" (LIU, 2010). 
A lei de energia renovável aprovada em 2005 no país permitiu uma nova etapa para o desenvolvimento da energia renovável, portanto, o governo chinês formulou uma série de politicas e leis para incentivar o seu uso, a saber: Subsídios do governo para alavancar a competitividade da produção de painéis; redução ou isenção de impostos, gerando assim motivação, entusiasmo, segurança e vantagens por parte do governo para os investidores o que o tem produzido investimento sólido na área.

Além de que "o governo fortalece a cadeia da indústria das células fotovoltaicas, especialmente focada na desenvoltura da tecnologia voltada para resoluções de problemas tais como falta da matéria prima do silício e seu alto custo incorporado" (WANG, 2010).

Nesse sentido, Wang (2010) reafirma que "o alto custo da geração da energia fotovoltaica é a maior barreira, contudo, fazem-se necessárias mudanças, bem como políticas que estimulem a grande e rica capacidade de produção do país".

\subsection{Paquistão}

O país é muito rico em irradiação solar, possibilitando o governo possuir 18 sistemas fotovoltaicos em várias partes do país; "O país do Paquistão é provido de quase todas as fontes de energias renováveis tais como: solar (térmica e fotovoltaica), eólica, biogás, hídrica, biomassa/ resíduos, geotérmica, marés, etc" (SHEIKH, 2009).

Infelizmente, por falta de gestão adequada essas plantas solares não existem mais; porque o país é ainda débil em organizações não governamentais, recursos humanos, incentivos técnicos e financeiros, impossibilitando assim, qualquer avanço em um futuro próximo.

Sheikh (2009) afirma que, "as principais dificuldades para sua utilização consistem no alto custo inicial do sistema, desconhecimento da população sobre tais benefícios, falta de conhecimento técnico, além do próprio governo não "enxergar" a necessidade da mesma".

“Através da politica nacional de energia renovável anunciada em 2002 foi possível projetar que os recursos energéticos renováveis adquirissem 3\% de participação no fornecimento de energia primária em 2010, além de alocação anual de $2 \%$ a favor do desenvolvimento de tais tecnologias" (KHAN, 2010).

\subsection{Malásia}

A Malásia possui um total de $20,5 \mathrm{GW}$ de capacidade instalada e a margem de reserva de sua península corresponde a mais $47 \%$, com uma média de $4 \%$ de crescimento anual; O país tem previsões de demanda de energia elétrica que chegam até 23,1GW em 2020, o que significa quase o dobro da demanda atual (OB, 2010).

A política energética implantada nas últimas três décadas tem demonstrado que o governo Malaio cuida do meio ambiente. Em 2005, a Malásia se comprometeu a reduzir 70 milhões de toneladas de Gás Carbônico em um período de 20 anos (LAU, 2009). 
De acordo com Lau (2009), "o lançamento da política e tecnologia baseada em eficiência energética renovável lançada em 2009 ofereceu a oportunidade de o país obter seu crescimento econômico". Esta política foi construída baseada em quatro pilares:

a) Alcançar a independência energética e promover a utilização eficiente;

b) Conservar e minimizar o impacto sobre o meio ambiente;

c) Melhorar a economia nacional através do uso da tecnologia;

d) Permitir que todos os Malaios desfrutem de uma melhor qualidade de vida.

Leis, regulamentos, incentivos econômicos, pesquisa e desenvolvimento, apoio técnico industrializado, projetos, etc, foram implantados no país, através de investimentos de US\$ 4 bilhões ao longo dos três últimos anos e criando assim, 11.000 empregos. Segundo Ob (2010), “o esquema poderia mudar a mentalidade dos consumidores, uma vez que as tarifas são baseadas no princípio do "poluidor- pagador"”.

Em relação à energia solar, o país tornou-se membro pleno e de direito do programa internacional de energia fotovoltaica (IEAPVPS) em 23 de outubro de 2008, todavia este mercado ainda é extremamente pequeno em comparação a outros países desenvolvidos (OB, 2010).

\subsection{Austrália}

O governo da Austrália fez com que a tarifa FIT fosse introduzida obrigatoriamente a fim de auxiliar a comercialização da energia renovável no país (PLAN, 2010). De acordo com Valentine (2010), "a tecnologia solar ainda é 3 a 4 vezes mais cara do que a energia elétrica a gás”.

Devido às mudanças climáticas os políticos australianos buscaram maneiras de reduzir o impacto de gases tóxicos na atmosfera; Por tal motivo, o país em 2001 tornou-se o primeiro a introduzir um mercado de energia renovável através de acordos certificados (ANDREWS, 2001). Apesar das politicas energéticas do país oferecer apoio significativo, as projeções recentes estimam energia solar fotovoltaica crescente a uma taxa anual de apenas 15MW até 2020 (CME, 2009).

\section{Considerações finais}

As buscas da redução dos impactos ambientais no setor de produção de energia elétrica veem ganhando proporções extremas perante as políticas e governo dos países em estudo. Os governos de cada país tem por objetivo incentivar um novo desenvolvimento industrial no setor de energia, promover o consumo consciente, reduzir desperdícios, custos, além de promover investimentos, e gerar assim, a eficiência energética. 
Segundo EPE (2012), “o sistema de tarifa-prêmio ("feed-in tariff'), adotado em quase todos os países da Europa, em que se observa expressiva instalação de sistemas fotovoltaicos conectados a rede, tem se revelado como o mais eficiente para incentivar o uso desta fonte".

A eficiência energética traz ganhos econômicos amplos, que segundo a Weo (2012), países como a Índia, China, Estados Unidos e na Europa já puderam notar. Contudo, fazem-se necessários investimentos em tecnologia, além de formular medidas, leis, normas, tarifas, incentivos, etc.

Nessa direção a Tabela 2 é apresentada para melhor visualização das políticas de energia solar dos países citados:

Tabela 2 - Políticas energéticas mundiais sobre o uso da energia solar

\begin{tabular}{|c|c|c|c|c|c|}
\hline PAÍS & Política & $\begin{array}{c}\text { Subsídio } \\
\text { governo }\end{array}$ & $\begin{array}{c}\text { Financiamento } \\
\text { / Empréstimo }\end{array}$ & $\begin{array}{c}\text { Suporte } \\
\text { a P\&D }\end{array}$ & $\begin{array}{c}\text { Capacidade } \\
\text { (instalada e/ou projeção) }\end{array}$ \\
\hline USA & RPS/ FIT & Sim & Sim & Sim & 25\% C. total em 2025 \\
\hline Canadá & FIT & Sim & Sim & Sim & 12 GW até 2016 \\
\hline Alemanha & FIT & Sim & Sim & Sim & 30 GW 2010 \\
\hline Espanha & FIT & Sim & Sim & Sim & 500 MW em 2010 \\
\hline França & FIT & Sim & Sim & Sim & Até 5,4 GW até 2020 \\
\hline China & & Sim & Sim & Sim & - \\
\hline Paquistão & & Sim & Sim & Sim & Gim 2020 \\
\hline Malásia & FIT & Sim & Sim & Sim & Sim \\
\hline Austrália & FIT & Sim & Sim & MW até 2020 \\
\hline
\end{tabular}

Fonte: Autoria própria (2013)

\section{Conclusão}

A energia solar é uma das fontes mais promissoras, consistentes e renováveis dentre todas as fontes de energia renováveis existentes, simplesmente porque ela é primária se renovando a cada dia, podendo ser explorada de várias maneiras, através das células fotovoltaicas, térmica, entre outras aplicações. Notadamente, quase todos os países que utilizam este tipo de energia, têm a necessidade de adotar políticas direcionadas e específicas para seu desenvolvimento.

Nessa direção, as tarifas $F I T$, isenção de impostos e outros tipos de subsídios determinados pelo poder público, são inseridos pelo governo em cada país, fazendo com que, o governo direcione forças para melhorar a qualidade do meio ambiente, desenvolvimento econômico, político e institucional. "Uma importante lição que justifica o relativo sucesso do "feed-in tariff” nestes países se deve fundamentalmente a atratividade deste instrumento segundo a ótica dos investidores (EPE, 2012). Políticas relacionadas ao uso de energia solar incluem histórias de sucesso em potências como EUA, Canadá, França, Espanha, China, Alemanha e Austrália. Tais países atestam que a utilização da energia solar pode ser uma medida viável de minimizar potencialmente os danos causados ao meio ambiente e a própria saúde humana em relação às energias provenientes de fontes não renováveis. 
Portanto, a energia gerada pelo sol é a forma mais primária de fonte energética, exemplo de energia renovável; Sua máxima utilização deve ser considerada por todos os países, igualmente a geração de políticas públicas, proporcionando o aumento de sua acessibilidade, direcionadas ao crescimento e desenvolvimento político, econômico, social e ambiental.

\begin{abstract}
With the intention to reduce dependence on fossil fuels, minimize environmental impacts and encourage new industrial development, the energy sector has caused strategic changes regarding their policy. The aim of this paper is to present a review of different energy policies on the use of solar energy in different countries in the world. The methodology used in this study was a literature search, which allowed the realization of a comparative analysis from a broad survey work. Energy policy is a strategy that governments address the development issues of energy consumption along with the industry to sustain its growth, including distribution, production and consumption; In this context, the global demand for primary energy is projected on a scenario to expand by almost $60 \%$ between 2002-2030. So, a FIT tariffs, tax exemptions and other subsidies determined by the public, are the most commonly used policies in emerging countries, since it, improve the quality of the environment, provides motivation for the development of significant interest and use of renewable energy technology, policy, institutional and mainly economic development.
\end{abstract}

Key-words: solar energy; energy policies; photovoltaic solar energy.

\title{
Referências
}

A CARTA DO SOL. Relatório técnico. Subsídios para o planejamento da promoção da energia solar fotovoltaica no brasil. rio de janeiro, 19 de agosto de 2011.

ADEME - Agence de l'Environnement et de la Maîtrise de l'Energie. Efficacité énergétique dans l'Union européenne : panorama des politiques et des bonnes pratiques. (2008). Disponível em < http://www2.ademe.fr/servlet/getDoc?cid=96\&m=3\&id=58124\&p1=00\&p2=08\&ref=17597\#>. Acesso em $17 \mathrm{de}$ fev. 2013.

ANDREWS, G. Market based instruments: Australia's experience with trading renewable certificates. Workshop on Good Practices in policies and measures, 8-10 October, Copenhagen; 2001.

ANEEL - Agencia Nacional de Energia Elétrica. Atlas de Energia Elétrica do Brasil. Ed.- Brasília: Cap.5- Outras Fontes, 2008.

BATTAGlini, A.; LILliESTAM, J.; HAAS, A., PATT, A. Development of SuperSmart Grids for a more efficient utilisation of electricity from renewable sources. Journal of Cleaner Production, v.17, 2009.

BHANDARI R, STADLER I. Grid parity analysis of solar photovoltaic systems in Germany using experiences curves. Solar Energy, v. 83, n. 9, p. $1634-44,2009$. cross ref

BRANKER K; PEARCE J. M. Financial return for government support of large-escala thin-film solar photovoltaic manufacturing in Canadá. Energy Policy, v. 38 n. 8, p. 4291-303, 2010. crossref

CARLEY S. State renewable energy electricity policies: na empirical evalution of effectiveness. Energy Policy, v. 37 , n. 8 , p. 3071-81, 2009. crossref

CEF, Canadian Energy Facts. Foreign Affairs and international Trade Canadá. Disponível em: <http://www.international.gc.ca/enviro/energy-energie/facts-faits.aspx?lang=eng; 2012>. Acesso em Nov. de 2012.

CME, Renewable Energy Projections: 2009-2028. Carbon market economics. Pty Ltd., Austrália, 2009. Disponível em: <http://www.aemo.com.au/planning/419-0032.pdf; 2009>. Acesso em Maio. 2013. 
DYSON, F. O Sol, o genoma e a internet. Companhia as letras, SP. 2001.

DW BRASIL. Disponível em <http://www.dw.de/produ\%C3\%A7\%C3\%.A-energia-solar-na-alemanha-baterecorde-em-2012/a-16492873> . Acesso em: 21 de abril de 2013.

DUSONCHET, L.; TELLARETTI, E. Economic analysis of different supporting policies for the production of electrical energy by solar photovoltaics in western European Union countries. Energy Policy; v. 38 n. 7, p. 3297-308, 2010. crossref

EPD. Energy policy definition. Disponível em: <http://www.tutorvista.com/ks/energy-policy-definition;2013>. Acesso em Fev de 2013.

EPE (Empresa de pesquisa energética)/ Nota técnica - Análise da Inserção da Geração Solar na Matriz Elétrica Brasileira. Rio de Janeiro. Maio de 2012.

ELKINS, P. Step changes for decarbonising the energy ststem: research needs for renewables, energy efficiency and nuclear power. Energy Policy, v. 32, p. 1891-904; 2004. cross ref

EREC. European Renewable Energy Council: Renewable energy target for Europe - 20\% by 2020. (Brief Paper, Brussel); 2005.

HASNAIN S.M; ELANI U.A; ALAWAJI S.H; ABAOUD H.A; SMIAL M.S. Prospects and proposals for solar energy education program. Apply Energy, v. 52, p. 307-14, 1995. cross ref

IEA, IEA-PVPS, Trends in photovoltaic applications. Survey Report of Selected IEA Countries between 1992 and 2007; 2008

KISSEL, M. K. S.C.W. Adaptations of renewable energy policies to unstable macroeconomics situations - case study: wind power in Brazil. Energy Policy, v 34, n. 18, p. 3591-8, 2006. crossref

KHAN, M. A.; LATIF N. Environmental friendly solar energy in Paquistan's scenario renewable sustainable. Energy Review; v. 14, n. 8, p. 2179-81, 2010.

LAU, L. C.; TAN, K. T.; MOHAMED, A. R. A comparative study on the energy policies in Japan and Malasya in fulfilling their nations obligations towards the Kyoto Protocol. Energy Policy, v. 37, n. 11, p. 4771-8, 2009. cross ref

LIMING, H.; HAQUE, E.; BARG, S. Public policy discourse, planning and measures toward sustainable energy strategies in Canada. Renewable Sustainable Energy Rev, v. 12, n. 1, p. 91-115, 2008. cross ref

LIU, L. Q.; WANG, Z. X.; ZHANG, H. Q.; XUE, Y. C. Solar energy development in china- a review. Renewable sustainable energy rev, v. 14, p. 301-11, 2010. crossref

LUIGI, D.; ENRICO, T. Economic analysis of different supporting policies for the productions of electrical energy by solar photovoltaics in western European Union countries. Energy Policy, v. 38, n. 7, p. 3297-308, 2010. cross ref

MDIC, MINISTÉRIO DO DESENVOLVIMENTO, INDÚSTRIA E COMÉRCIO EXTERIOR. Oportunidade de negócios em serviços Brasil Espanha. Brasil, 2007.

OB, T.H.; P, S.Y.; C, S.C. Energy policy and alternative energy in Malasya: issues and challenges for sustainable growth. Renewable Sustainable Energy Rev, v. 14, n. 4, p. 1241-52, 2010. crossref

PLAN, S. Australian government department of climate change of energy efficiency, 2010.

SARAH, J. B.; L, H.R. Nodal pricing in Otário, Canadá: implications for solar PV electricity. Renewable Energy, v. 34 , p. $170-8,2009$. crossref

SCHETTINO, S. Cenários do uso das redes elétricas inteligentes (smart grid): tendências de sua difusão no Brasil. Dissertação em Engenharia de Produção/ Stevon Schettino - João Pessoa: UFPB, 2013.

SILVA, R. M. Um Modelo para Análise da Sustentabilidade de Fontes Elétricas - Tese de doutorado em Administração, da Universidade Federal de Pernambuco, CCSA, Programa de Pós Graduação em Administração PROPAD, Recife, 2011. 
SHEIKH, M.A. Renewable energy resource potencial in Pakistan. Renewable Sustainable Energy Rev, v. 13, n. 9, p. 2696-702, 2009. crossref

SOLANGI, K.H.; ISLAM, M.R.; SAIDUR, R.; RAHIM, N.A.; FAYAZ, H. A review on global solar energy policy. Renewable and Susteinable Energy Reviews, v. 15, p. 2149-2163, 2011. crossref

SOUZA, F.R. Impacto do Preço do Petróleo na Política Energética Mundial. (COPPE/UFRJ, M.Sc., Planejamento Energético, 2006). Dissertação em Engenharia de Produção- Universidade Federal do Rio de Janeiro, COPPE. XI, 160 p. 29; 2009.

TSOUTOS, T.; FRANTZESKAKI, N.; GEKAS, V. Environmental impacts from the solar energy Technologies. Energy Policy, v. 33, n. 3, p. 289-96, 2005. crossref

VALENTINE, S. Braking wind in Australian: a critical evaluation of the renewable energy target. Energy Policy, v. 38, n. 7, p. 3668-75, 2010. crossref

VIANA, T. S; RUTHER, R.; MARTINS, F. R.; PEREIRA, E.B. Assessing the potencial of concetrating solar photovoltaic generation in Brazil with satélite- derived direct normal irradiation. Solar Energy, v. 85, p. 486-495, 2011. crossref

WANG, Q. Effective policies for renewable energy - the example of China's wind power- lesson for China's photovoltaic power. Renewable Sustainable Energy Rev, v. 14, n. 2, p. 702-12, 2010. crossref

WEO, World Energy Outlook, 2012. Presentation to the press London, 12 november 2012. Disponível em: <http://www.worldenergyoutlook.org/pressmedia/recentpresentations/PresentationWEO2012launch.pdf>. Acesso em 23 de abril 2013.

\section{Dados dos autores}

Nome completo: Adriana de Souza Nascimento

Filiação institucional: Universidade Federal da Paraíba - UFPB

Função ou cargo: Mestranda em Engenharia de Produção

Endereço completo para correspondência: Av. Mar Cáspio, 367. Apt. 103. Edf. Canaã II.

Intermares. Cabedelo - PB - Brasil. Cep: 58310000.

Telefone para contato: 55-83-8837 8771

e-mail: adriana.souza.adm@hotmail.com

Nome completo: Ricardo Moreira da Silva

Filiação institucional: Universidade Federal da Paraíba - UFPB

Função ou cargo: Professor

Endereço completo para correspondência: Universidade Federal da Paraíba, Centro de Tecnologia -

Campus I. Departamento de Engenharia de Produção. Cidade Universitária. Cep: 58000-000.

João Pessoa, PB - Brasil.

Telefone para contato: 55 - 83 - 87550155

e-mail: ricardomoreira0203@hotmail.com

Nome completo: Eufrásio Vieira dos Anjos Júnior 
Filiação institucional: Universidade Federal da Paraíba - UFPB

Função ou cargo: Mestrando em Engenharia de Produção

Endereço completo para correspondência: Rua Celso Cirne, 211. Solânea - PB. Cep: 58225-000 Brasil.

Telefone para contato: 55- 83 - 9802-5915

e-mail: eufrasiovieira@hotmail.com

Submetido em: 16/10/2013

Aceito em: 22/10/2014 